Cite this: Phys. Chem. Chem. Phys.,

Received 14th June 2016, Accepted 11th July 2016

DOI: $10.1039 / c 6 c p 04146 a$

www.rsc.org/pccp

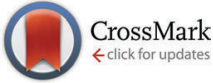
$2016,18,21527$

\section{Picosecond to nanosecond dynamics provide a source of conformational entropy for protein folding}

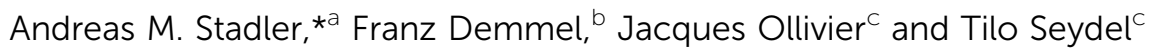

Myoglobin can be trapped in fully folded structures, partially folded molten globules, and unfolded states under stable equilibrium conditions. Here, we report an experimental study on the conformational dynamics of different folded conformational states of apo- and holomyoglobin in solution. Global protein diffusion and internal molecular motions were probed by neutron time-of-flight and neutron backscattering spectroscopy on the picosecond and nanosecond time scales. Global protein diffusion was found to depend on the $\alpha$-helical content of the protein suggesting that charges on the macromolecule increase the short-time diffusion of protein. With regard to the molten globules, a gel-like phase due to protein entanglement and interactions with neighbouring macromolecules was visible due to a reduction of the global diffusion coefficients on the nanosecond time scale. Diffusion coefficients, residence and relaxation times of internal protein dynamics and root mean square displacements of localised internal motions were determined for the investigated structural states. The difference in conformational entropy $\Delta S_{\text {conf }}$ of the protein between the unfolded and the partially or fully folded conformations was extracted from the measured root mean square displacements. Using thermodynamic parameters from the literature and the experimentally determined $\Delta S_{\text {conf }}$ values we could identify the entropic contribution of the hydration shell $\Delta S_{\text {hydr }}$ of the different folded states. Our results point out the relevance of conformational entropy of the protein and the hydration shell for stability and folding of myoglobin.
\end{abstract}

\section{Introduction}

Protein folding is a fundamental process in molecular biology. The protein folding transition occurs from the fully unfolded peptide chain via metastable and partially folded intermediates towards the stable and native folded structure at the end of the folding collapse. ${ }^{1}$ In the nucleation-condensation model of protein folding the cooperative formation of secondary and tertiary structures is a key aspect, ${ }^{2}$ while in the diffusioncollision model the diffusion of micro-domains and their collision have central importance for protein folding kinetics. ${ }^{3}$ This illustrates that motions in proteins are highly relevant for protein folding. From a thermodynamic point of view, it is the difference in the free energy $\Delta G$ between the unfolded state and the folded state $\left(\Delta G=\Delta G_{\text {unfolded }}-\Delta G_{\text {folded }}=\Delta H-T \Delta S\right)$ that drives the folding transition along the folding pathway. Weak van der Waals forces, hydrogen bonds, and, to a smaller

\footnotetext{
${ }^{a}$ Jülich Centre for Neutron Science JCNS and Institute for Complex Systems ICS, Forschungszentrum Jülich GmbH, 52425 Jülich, Germany. E-mail: a.stadler@fz-juelich.de

${ }^{b}$ ISIS Facility, Rutherford Appleton Laboratory, Didcot OX11 OQX, UK

${ }^{c}$ Institut Laue-Langevin, 71 Avenue des Martyrs, CS 20156, 38042 Grenoble Cedex 9, France
}

extent, screened electrostatic interactions contribute to the enthalpic stabilization $\Delta H$ in proteins, ${ }^{4}$ whereas the entropic term $T \Delta \mathrm{S}$ contains contributions both from the protein $\Delta S_{\text {conf }}$ and from the hydration shell $\Delta S_{\text {hydr }}$. Internal protein dynamics contribute importantly to the conformational entropy $\Delta S_{\text {conf }}$ of the macromolecule as well as to the sampling of different conformations during the folding process.

Localized fast diffusive motions of amino-acid side-chains provide a source of conformational entropy $\Delta S_{\text {conf }}$ of the macromolecule. ${ }^{5}$ The conformational entropy content of the amino-acid side-chains depends on the degree of folding and on the compactness of the local environment. ${ }^{6}$ Therefore, amino-acid side-chain dynamics are relevant for the entropic stabilization of the unfolded states as compared to the folded native protein conformation. Motions in proteins are known to span a wide time range from the femtosecond of fast bond vibrations to the millisecond of slow and large domain motions. ${ }^{7}$ Protein side-chain dynamics, however, take place mostly on the pico- to nanosecond time scale. ${ }^{8}$ Internal localized protein dynamics are not limited to fast side-chain motions, but extend into the nanosecond time scale for slow motions of the protein backbone or loops, ${ }^{7}$ or of flexible and disordered regions. ${ }^{9}$ Apart from internal dynamics, proteins in solution also undergo 
global protein diffusion. Global diffusion depends strongly on the size and shape of the protein as well as on interactions with the environment. In dilute solution, protein-protein interactions are negligible, but are relevant for macromolecular diffusion at higher concentrations.

Myoglobin (Mb) serves as a model system in the field of biophysics ${ }^{10}$ as it is comparatively small, which allows us to obtain an understanding of the underlying physical principles governing its dynamical behaviour, and it is still sufficiently complex to show typical protein behaviour. In the following context, the heme-bound protein is termed holo-Mb. Apomyoglobin (apo-Mb) - myoglobin without the heme group - is less stable than holo-Mb and can be trapped in different folded, partially folded molten globules (MGs) and unfolded states under equilibrium conditions depending on the solvent conditions chosen. ${ }^{11-13}$ The structural and dynamic properties of the different equilibrium folding states of apo-Mb have been studied by NMR spectroscopy, ${ }^{14-17}$ SAXS ${ }^{18,19}$ and also by FRET. $^{20}$ In our recent work, we have investigated the fast internal dynamics of apo- and holo-Mb on the time scale of a few picoseconds using neutron spectroscopy ${ }^{21}$ However, further information concerning the internal dynamics of different folded states on the slower picosecond to nanosecond time scale and the properties of global protein diffusion as a function of the degree of folding is still missing.

Quasielastic incoherent neutron spectroscopy (QENS) is a wellsuited technique to measure the average dynamics of biological macromolecules on the picosecond to the nanosecond time scale. ${ }^{22}$ QENS measurements of protein solutions performed on neutron spectrometers with high-energy resolution allow us to determine internal protein dynamics and global protein diffusion simultaneously. Mostly, proton dynamics are observed by QENS due to the large incoherent scattering cross section of ${ }^{1} \mathrm{H}$ compared to all other chemical elements. As protons are uniformly distributed in proteins, QENS probes average protein dynamics. On the accessible time scale of QENS, protons act as reporters for the dynamics of chemical groups to which they are bound covalently.

In this article, we report on a comparative experimental study on the dynamics of different folded structural states of holo- and apo-Mb as a function of temperature that extends our previous investigation on the fast picosecond dynamics. ${ }^{21} \mathrm{We}$ used high-resolution neutron time-of-flight and backscattering spectroscopy to focus on slower picosecond to nanosecond dynamics. The samples have been measured in the solution state to allow for solvent induced effects and to enable reversible thermodynamic properties. Global protein diffusion and internal macromolecular dynamics could be separated from the recorded QENS spectra. Detailed insight into the properties of the internal dynamics of different folded states of the protein was obtained from the recorded data.

\section{Material and methods}

\subsection{Sample preparation}

$\mathrm{Mb}$ from equine skeletal muscle was obtained commercially (product number M0630, Sigma Aldrich). Apo-protein was obtained by using the butanone method as described in the previous work. ${ }^{21}$ To remove exchangeable protons, the dry protein powders were dissolved in $\mathrm{D}_{2} \mathrm{O}$ (99.9\% atom D, Sigma Aldrich), incubated for one day and lyophilized afterwards. The $\mathrm{D}_{2} \mathrm{O}$ exchanged protein powders were then dissolved in different $\mathrm{D}_{2} \mathrm{O}$ buffers ((i and ii) $20 \mathrm{mM} \mathrm{NaH} \mathrm{PO}_{4} / \mathrm{Na}_{2} \mathrm{HPO}_{4}$ at pD 4 and 6; (iii) $10 \mathrm{mM}$ DCl at pD 2; (iv) $10 \mathrm{mM}$ DCl, $20 \mathrm{mM}$ NaTCA at pD 2; (v) $10 \mathrm{mM}$ $\mathrm{DCl}, 100 \mathrm{mM} \mathrm{NaCl}$ at pD 2) at a concentration of approximately $5 \mathrm{mg} \mathrm{mL}{ }^{-1}$ and dialyzed against the corresponding $\mathrm{D}_{2} \mathrm{O}$ buffer in excess volume (Slide-A-Lyzer dialysis devices, $3500 \mathrm{MWCO}$, Thermo Scientific, Rockford, IL). The protein solutions were concentrated to the concentrations used for neutron scattering experiments (Vivaspin 3000 MWCO concentration units, Sartorius, Goettingen, Germany). The pD of the filtered $\mathrm{D}_{2} \mathrm{O}$ buffer was measured to verify whether the correct $\mathrm{pD}$ value has been achieved in the concentrated protein solution. The $\mathrm{pD}$ was calculated by adding 0.4 to the value measured on a normally calibrated $\mathrm{pH}$ meter. ${ }^{23}$ Deuterated DCl and NaOD (99 atom\%, Sigma-Aldrich) were used for pD adjustment of the $\mathrm{D}_{2} \mathrm{O}$ buffers.

The protein concentration was measured using $\mathrm{UV} / \mathrm{vis}$ absorption spectroscopy (Nano-Drop 2000c, Thermo Scientific). The concentration of the apo-Mb solutions was determined using the calculated extinction coefficient $E_{1 \%}=8.25$ at $280 \mathrm{~nm}$ from the amino acid sequence using the ExPASy web server. ${ }^{24} \mathrm{Mb}$ was found to be in the oxygenated state, and the concentration was determined using extinction coefficients of 13.9 and $14.4 \mathrm{mM}^{-1} \mathrm{~cm}^{-1}$ at 542 and $579 \mathrm{~nm}$, respectively, and a molecular mass of $18.8 \mathrm{kDa}$ as reported by Antonini and Brunori. ${ }^{25}$

\subsection{QENS experiments}

The neutron scattering experiments were performed on the disc chopper neutron time-of-flight spectrometer $\mathrm{IN}^{26}$ at the Institut Laue-Langevin (ILL) in Grenoble, France, on the neutron backscattering spectrometer IRIS ${ }^{27}$ at the ISIS spallation source at the Rutherford Appleton Laboratory in Oxfordshire, UK, and on the high-resolution neutron backscattering spectrometer IN16 at the ILL. ${ }^{26}$ On IN5, the incident wavelength was set at $10 \AA$ and a chopper speed of $12000 \mathrm{rpm}$ combined with a chopper ratio of $5 / 6$ was used, which yielded an energy resolution of approximately $12 \mu \mathrm{eV}$ (full-width half-maximum FWHM). Data recorded on IN5 were analysed in the scattering vector range between 0.29 and $1.10 \AA^{-1}$ and in the energy transfer range between -2 and $0.5 \mathrm{meV}$. The IRIS spectrometer is characterized by an energy resolution of $18 \mu \mathrm{eV}$ (FWHM). Data recorded on IRIS were analysed between 0.5 and $1.49 \AA^{-1}$ and between -0.5 and $0.5 \mathrm{meV}$. The energy resolution of the neutron backscattering spectrometer IN16 is $0.9 \mu \mathrm{eV}$ (FWHM). IN16 data were analysed between 0.19 and $0.88 \AA^{-1}$ and between -14.1 and $14.1 \mu \mathrm{eV}$. Resolution functions were measured using a $1 \mathrm{~mm}$ thick vanadium slab. The individual spectra of the neutron detectors were grouped and energy channels were binned for better statistics for all instruments. Measured intensities were corrected for energy-dependent neutron detector efficiency, normalized to vanadium, and transformed into energy transfer and scattering vector space. The measured intensities of the samples were divided by the calculated sample transmissions. Multiple scattering 
corrections were not performed, as the transmissions of all samples were above or close to 0.9. All samples were mounted in flat $\mathrm{Al}$ cells with $1 \mathrm{~mm}$ thickness and were oriented at $135^{\circ}$ with respect to the incident beam. The sample holders were sealed with In wire against vacuum. All protein samples and buffer solutions received around $4 \mathrm{~h}$ neutron beam time on IN5, $8 \mathrm{~h}$ on IRIS and $24 \mathrm{~h}$ on IN16 per temperature point.

For the QENS spectra of the protein solutions measured on IN5 and IRIS the $\mathrm{D}_{2} \mathrm{O}$ buffers make a notable contribution. Therefore, the measured spectra of the $\mathrm{D}_{2} \mathrm{O}$ buffers were subtracted from the spectra of the protein solutions according to

$$
S(q, \omega)=S(q, \omega)_{\text {protein solution }}-(1-\phi) \cdot S(q, \omega)_{\text {buffer }},
$$

where $\phi=\bar{v} \cdot c$ is the volume fraction of the protein with the partial specific volume $\bar{v}$ of the protein and the measured protein concentration $c$. The partial specific volume of proteins is typically in the range between 0.70 and $0.75 \mathrm{~mL} \mathrm{~g}^{-1},{ }^{28}$ with an experimentally determined average value of $0.73 \mathrm{~mL} \mathrm{~g}^{-1} \cdot{ }^{29}$ The measured value of $\mathrm{Mb}$ is $\bar{v}=0.74 \mathrm{~mL} \mathrm{~g}^{-1},{ }^{30}$ which is close to the reported experimental average value. For the subtraction of the $\mathrm{D}_{2} \mathrm{O}$ buffers we used the reported value of $\mathrm{Mb}$, but the resulting protein spectra are not modified within the error bars when the average value is taken instead.

With regard to the data measured on IN16, the contribution of the empty Al sample holder was subtracted from the protein solutions as the $\mathrm{D}_{2} \mathrm{O}$ buffers appear only as a flat background in the limited energy transfer window. The $\mathrm{D}_{2} \mathrm{O}$ contribution was accounted for during data analysis with a linear background.

\subsection{QENS data analysis}

A description of the QENS technique can be found in the book by Bée. ${ }^{31}$ The application of QENS for the study of biomolecular dynamics is described in the book by Fitter, Gutberlet and Katsaras. $^{22}$

The QENS spectra of internal protein dynamics can be described by a model independent approach according to ${ }^{22}$

$$
S_{\mathrm{I}}(q, \omega)=A_{0}(q) \times \delta(\omega)+\left[1-A_{0}(q)\right] \times L_{\mathrm{I}}(q, \omega),
$$

where $A_{0}(q)$ is the elastic incoherent structure factor (EISF), which contains detailed information on internal protein motions that are confined with respect to the length-time window of the spectrometer. The Lorentzian $L_{\mathrm{I}}(q, \omega)$ effectively describes the quasielastic broadening caused by internal diffusive motions in the protein. It has the form of

$$
L_{\mathrm{I}}(q, \omega)=\frac{1}{\pi} \cdot \frac{\Gamma_{\mathrm{I}}(q)}{(\hbar \omega)^{2}+\Gamma_{\mathrm{I}}(q)^{2}},
$$

where $\Gamma_{\mathrm{I}}(q)$ are the half-widths at half-maximum (HWHM).

Within the accessible time- and length scale of neutron backscattering and time-of-flight spectrometers, the combination of translational and rotational diffusion of proteins in solution can be described effectively using one Lorentzian ${ }^{32-36}$

$$
S_{\mathrm{G}}(q, \omega)=\frac{1}{\pi} \cdot \frac{\Gamma_{\mathrm{G}}(q)}{(\hbar \omega)^{2}+\Gamma_{\mathrm{G}}(q)^{2}} .
$$

The theoretical scattering function for proteins in solution is the convolution of eqn (2) and (4) yielding

$$
S(q, \omega)=\left\{A_{0}(q) \times L_{\mathrm{G}}(q, \omega)+\left[1-A_{0}(q)\right] \times L_{\mathrm{G}+\mathrm{I}}(q, \omega)\right\},
$$

where

$$
L_{\mathrm{G}+\mathrm{I}}(q, \omega)=\frac{1}{\pi} \cdot \frac{\Gamma_{\mathrm{G}}(q)+\Gamma_{\mathrm{I}}(q)}{(\hbar \omega)^{2}+\left[\Gamma_{\mathrm{G}}(q)+\Gamma_{\mathrm{I}}(q)\right]^{2}} .
$$

The scattering function $S(q, \omega)$ plus linear background $B(\omega)$ was convoluted numerically using the measured instrumental resolution function $S_{\text {res }}(q, \omega)$ and fitted to each measured spectra $S(q, \omega)_{\text {meas }}$ according to

$$
S_{\text {meas }}(q, \omega)=\left[\exp \left(-\left\langle x_{\mathrm{vib}}{ }^{2}\right\rangle q^{2}\right) \times S(q, \omega)+B(\omega)\right] \otimes S_{\text {res }}(q, \omega) .
$$

The mean square displacements of fast vibrational motions $\left\langle x_{\mathrm{vib}}{ }^{2}\right\rangle$ were taken into account by a Debye-Waller factor.

\section{Results and discussion}

\subsection{QENS experiments with protein solutions}

A compilation of the different samples investigated by QENS in this study is given in Table 1 . The $\alpha$-helical content of the samples in heavy water buffer was measured by circular dichroism and has been reported before. ${ }^{21}$ Depending on the solvent composition, unfolded, partially folded MGs, or folded conformations can be stabilized under equilibrium conditions. On the high-resolution instrument IN16, all the listed conformational states were measured at concentrations of $5-6 \%(\mathrm{w} / \mathrm{v})$ at a uniform temperature of $289.2 \mathrm{~K}$. The high-energy resolution $\Delta E=0.9 \mu \mathrm{eV}$ of IN16 allows probing slow dynamics on a timescale up to approximately $\Delta t=1 \mathrm{~ns}(\Delta t=\hbar / \Delta E)$. On IRIS and IN5, temperature-dependent QENS experiments have been performed on the acid denatured state of apo-Mb, a MG state and a folded conformation, either the fully folded apo-Mb or the heme-bound holo-Mb. The energy resolutions of IRIS and IN5 are 18 and $12 \mu \mathrm{eV}$ corresponding to the slowest observable motions up to approximately $\Delta t=40$ and $60 \mathrm{ps}$, respectively. Higher protein concentrations have been measured on IRIS (6-8\%) to reduce the required neutron beam time per sample, while on IN5 lower protein concentrations (3-5\%) could be used due to the higher incident neutron flux and the large detector of the instrument. We would like to mention that the protein solutions of partially folded apo-Mb in the MG states had a

Table $1 \alpha$-Helical content measured by circular dichroism, ${ }^{21}$ unfolded (U), folded (F), molten globule (MG) states, solvent composition and protein concentration of the protein solutions investigated

\begin{tabular}{llllll}
\hline $\begin{array}{l}\alpha \text {-Helical } \\
\text { content (\%) }\end{array}$ & State & $\begin{array}{l}\text { Solvent } \\
\text { composition }\end{array}$ & $\begin{array}{l}c(\text { IN16) } \\
\left(\mathrm{mg} \mathrm{mL}^{-1}\right)\end{array}$ & $\begin{array}{l}c \text { (IRIS) } \\
\left(\mathrm{mg} \mathrm{mL}^{-1}\right)\end{array}$ & $\begin{array}{l}c(\mathrm{IN5}) \\
\left(\mathrm{mg} \mathrm{mL}^{-1}\right)\end{array}$ \\
\hline 6 & $\mathrm{U}$ & $\begin{array}{l}\text { apo-Mb pD 2 } \\
\text { apo-Mb pD 2, }\end{array}$ & 52 & 65 & 41 \\
22 & MG & $\begin{array}{l}\text { apo-1 M NaCl } \\
\text { apo-Mb pD 4 }\end{array}$ & 54 & - & - \\
43 & MG & $\begin{array}{l}\text { apo-Mb pD 6 } \\
\text { apo-Mb }\end{array}$ & - & 32 \\
64 & F & holo-Mb pD 6 & 56 & 56 & 48
\end{tabular}


gel-like viscosity at $5 \%$ or above. The MGs remained completely liquid at a concentration of $3 \%$.

A selection of representative QENS spectra of unfolded, partially folded MGs and folded conformations of apo- and holo-Mb that have been measured on the instrument IN16 are given in Fig. 1. In Fig. 2, exemplary QENS spectra of unfolded acid denatured apo-Mb measured on IRIS and IN5 at small and large scattering vectors are shown. The QENS spectra of the protein solutions after buffer subtraction were analysed according to eqn (7) containing one narrow Lorentz function for global
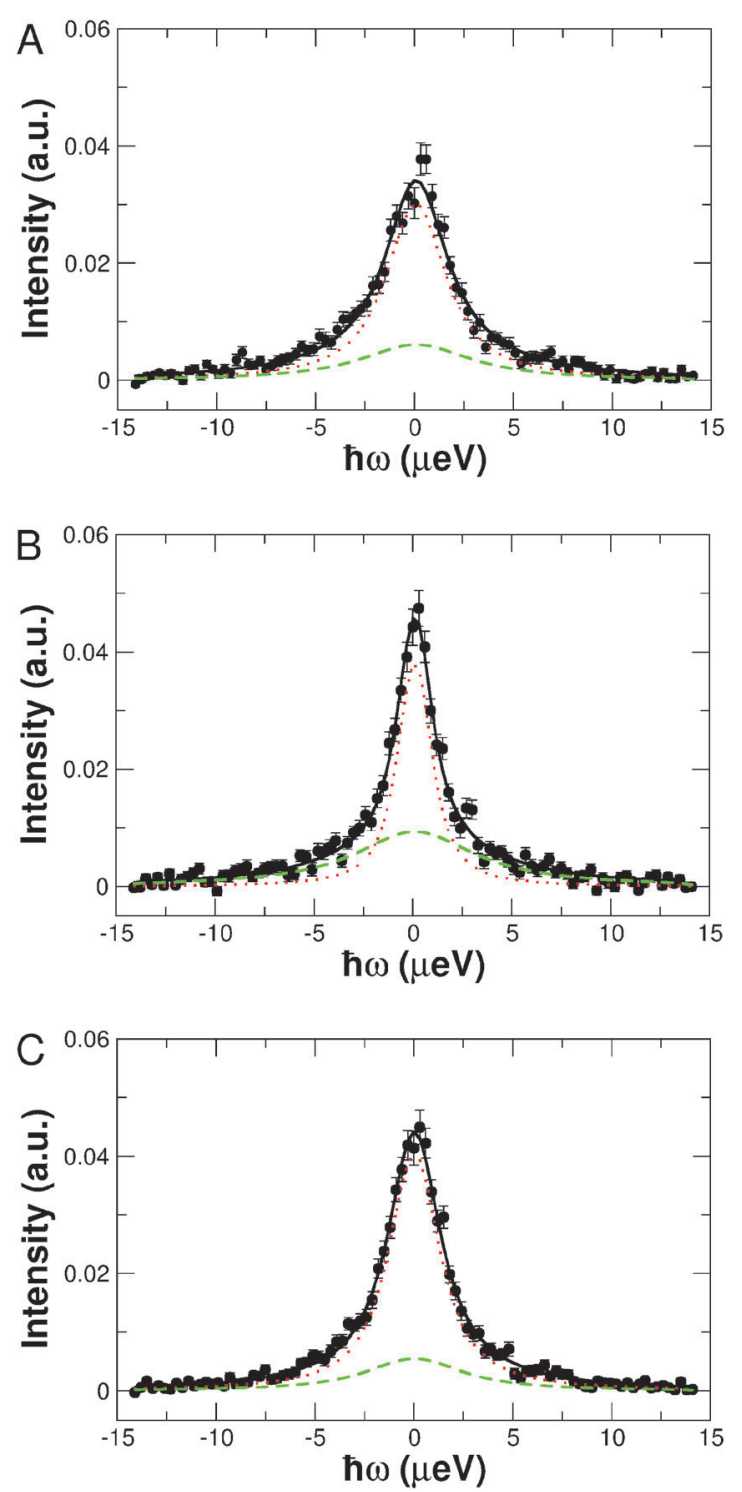

Fig. 1 QENS spectra of apo- and holo-Mb in different folded states measured on IN16 at a scattering vector of $q=0.65 \AA^{-1}$. (A) Acid denatured apo-Mb at pD 2 (6\% $\alpha$-helical content), (B) MG of apo-Mb at pD 4 (43\% $\alpha$-helical content), and (C) native folded structure of holo-Mb at pD 6 (66\% $\alpha$-helical content). The solid black line is the total fit to the spectra. The red dotted line is a narrow Lorentzian for global protein diffusion and the green dashed line a broad Lorentzian for the combination of internal and global protein dynamics. The curves are convoluted with the instrumental resolution. protein diffusion and one broad Lorentzian to account for the combination of global diffusion and internal diffusive motions in the protein. The used theoretical model provides a good description of the measured QENS data of all investigated samples measured on all the spectrometers used. In the following sections, we present and discuss the results obtained by using this simplified standard model ${ }^{32,33}$ (and references therein). We begin with the section concerning global protein diffusion.

\subsection{Global protein diffusion}

The line-widths $\Gamma_{1}$ of the narrow Lorentzian contain the relevant information about global protein diffusion. The dependence of $\Gamma_{1}(q)$ as a function of the scattering vector $q$ of acid denatured unfolded apo-Mb at pD 2 ( $6 \% \alpha$-helical content) measured on all the neutron spectrometers used, is shown in Fig. 3, as an illustrative case. The line-widths of all measured samples show the expected behaviour of $\Gamma_{1}(q)=D_{\text {eff }} \cdot q^{2}$ with the effective diffusion coefficient $D_{\text {eff }}$ that describes the combination of translational and rotational protein diffusion. Each spectrum was fitted independently and no $D_{\text {eff }} q^{2}$ behaviour was imposed for the fit. This further corroborates the robustness of the results. It has been shown that on the time-length window of neutron time-of-flight and backscattering spectroscopy, rotational diffusion yields $D_{\text {eff }}$ values that are about $30 \%$ larger than pure translational centre-of-mass diffusion. ${ }^{32-36}$ The shape of the QENS spectra due to the combination of rotational and translational diffusion remains Lorentzian with a linear increase of $\Gamma_{1}(q)$ versus $q^{2} \cdot{ }^{32-36}$ The line-widths measured on IRIS and IN5 at the lowest temperatures deviate from the linear behaviour for $q^{2} \rightarrow 0$. The explanation for this behaviour is the comparatively large energy resolution of IRIS and IN5, which does not allow resolving very narrow line-widths in the range of only a few $\mu \mathrm{eV}$ accurately.

The effective diffusion coefficients measured on IRIS and IN5 as a function of temperature are shown in Fig. 4(A) for the different folded conformations. In the investigated small temperature range, we find a linear increase of the diffusion coefficients with temperature as expected for a Stokes-Einstein temperature dependence. This also means that the proteins do not significantly change the effective volume they occupy in this regime. However, the $D_{\text {eff }}$ of apo-Mb at pD 6 (54\% $\alpha$-helical content) deviate from linearity at 310 and $320 \mathrm{~K}$. An explanation for this behaviour is the onset of protein aggregation or oligomerisation at higher temperatures, which reduces global protein diffusion. All samples were prepared in our lab in Jülich a few days before the neutron scattering experiments, and the apo-Mb sample (at pD 6) was measured on the last day of the experiment. The aging process might have contributed to the aggregation tendency at higher temperatures. It is already evident from Fig. 4(A) that there is a systematic decrease in $D_{\text {eff }}$ with the proteins being more folded for data recorded on both spectrometers. To allow for a quantitative and facilitated comparison of the results obtained from the spectrometers used, the $D_{\text {eff }}$ values determined on IRIS and IN5 were linearly interpolated to $289.2 \mathrm{~K}$ and are plotted in Fig. 4(B) together with the $D_{\text {eff }}$ determined on IN16 at $289.2 \mathrm{~K}$. 

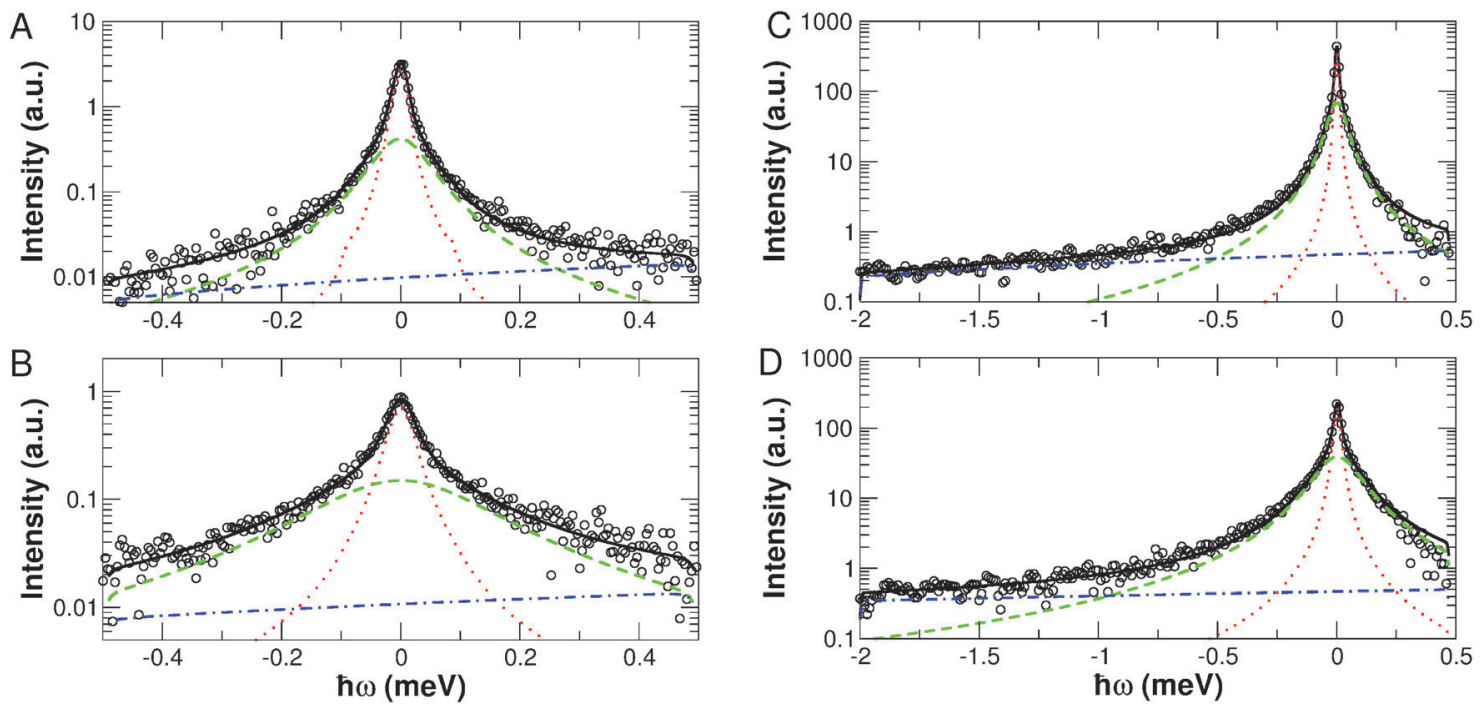

Fig. 2 QENS spectra of unfolded apo-Mb at pD $2\left(6 \% \alpha\right.$-helical content) measured on IRIS (A) at $q=0.50 \AA^{-1}$ and (B) $q=1.44 \AA^{-1}$, and on IN5 (C) at $q=0.54 \AA^{-1}$ and (D) $q=1.10 \AA^{-1}$. The sample temperatures are $303 \mathrm{~K}$ and $295 \mathrm{~K}$ for the shown IRIS and IN5 data, respectively. Empty symbols are the experimental data. For clarity, only every fourth data point is shown for IRIS spectra and every fifth point for data measured on IN5. The solid black line represents the total fit to the QENS spectra, the red dotted line is the narrow Lorentzian accounting for global protein diffusion, the green dashed line is the broad Lorentzian caused by the combination of global diffusion and internal protein dynamics, and the blue dashed-dotted line accounts for the linear background. All curves are convoluted using the instrumental resolution functions.

In the following section, we focus our discussion on the properties of the global effective diffusion coefficients at $289.2 \mathrm{~K}$ as shown in Fig. 4(B). Due to the lower protein concentrations of acid denatured apo-Mb at pD $2(c=3 \%)$ and of the MG state at pD $4(c=4 \%)$ measured on IN5, the diffusion coefficients determined are larger than the values of the other samples investigated on IRIS and IN5, and remain at a similar level irrespective of the degree of $\alpha$-helical content. The reason here is that hydrodynamic interactions slow down global protein diffusion at higher protein concentration. ${ }^{37}$ In the long-time limit of global protein diffusion observed by dynamic light scattering, unfolded and MG states of apo-Mb are characterized by slower protein diffusion due to the larger structural expansion as compared to the native folded states. ${ }^{21}$ On the other hand, the short-time limit of global protein diffusion probed on IRIS and IN5 follows an inverse behaviour for proteins at higher concentration $(c \geq 5 \%)$ : a reduced diffusivity with the increasing degree of $\alpha$-helical content. A physical explanation for this behaviour would be that repulsive interactions between charged proteins at $\mathrm{pD} 2$ and 4 at high concentrations increase protein diffusion in the short-time limit. Indeed, charged colloidal spheres diffuse slightly faster than non-charged spheres at the volume fractions explored here. ${ }^{37}$ At lower protein concentration $(c=3-4 \%)$ charge interactions seem to play a minor role.

With regard to the $D_{\text {eff }}$ measured on IN16, we obtain reduced absolute values as compared to the IRIS and IN5 results. Global protein diffusion of acid denatured apo-Mb is slightly faster than the folded conformations, which is in agreement with the observations described above. We find the $D_{\text {eff }}$ value of holo-Mb measured on IRIS being a factor of 1.64 larger than the corresponding IN16 result. After rescaling, the $D_{\text {eff }}$ values of acid denatured and folded apo-Mb determined on the low-resolution spectrometers coincide with the IN16 results (see the dotted line in Fig. 4(B)). The explanation here is that the slow internal process in the protein (vide infra) interferes with the narrow Lorentzian in the QENS spectra measured on the lower-resolution instruments, which cannot discriminate both processes accurately due to the lower energy resolution. As a result, larger effective diffusion coefficients are obtained on IRIS and IN5.

A further remarkable observation is that the diffusivity of the MG states measured on the high-resolution spectrometer is strongly reduced as compared to the other protein solutions. We would like to remind that the samples of the MG states had gel-like properties. Previously, it was observed by high-resolution neutron spectroscopy of concentrated protein solutions around the denaturing temperature that protein denaturation results in the formation of an entangled gel-like network with significantly reduced global protein diffusion as compared to the native folded state. ${ }^{38}$ With regard to the MG states the reduced $D_{\text {eff }}$ values also seem to be the signature for the onset of protein entanglement of the partially folded proteins on the nanosecond time scale. Although the acid denatured state is even fully unfolded, the higher charge of the protein increases protein repulsion, which appears to prevent protein entanglement and the reduction of global protein diffusion. With regard to the MG state with $22 \% \alpha$-helical content we find a significant discrepancy between the scaled IRIS and IN16 data. That observation indicates that on the faster picosecond time scale probed on IRIS the initial step of global protein diffusion is not sensitive to protein entanglement, while on the slow nanosecond time scale as observed on IN16 the individual proteins sense the presence of the neighbouring macromolecules, which reduces global protein diffusion. 

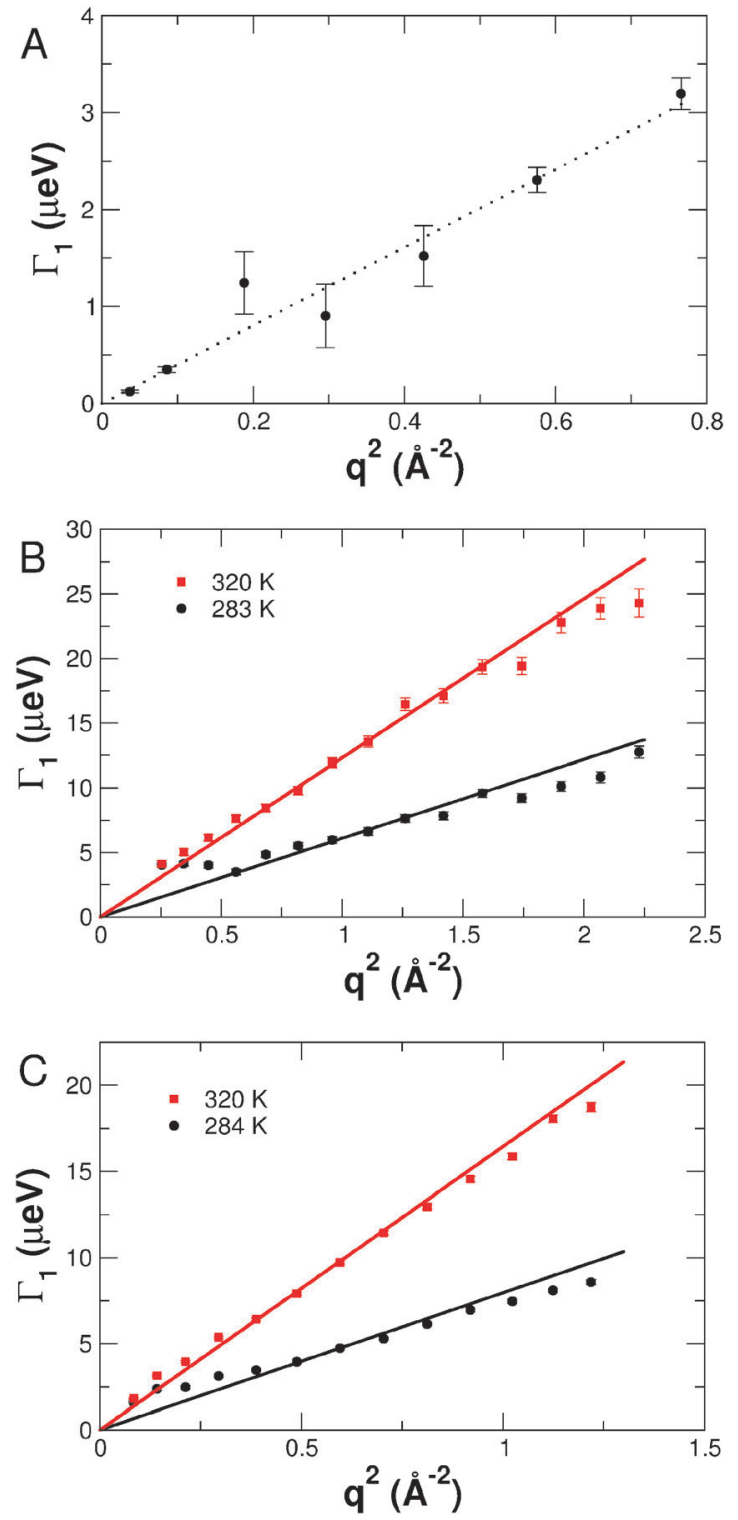

Fig. 3 Line-widths $\Gamma_{1}$ of the narrow Lorentzian describing global protein diffusion of the acid denatured unfolded apo-Mb at pD 2 (6\% $\alpha$-helical content) measured on the neutron spectrometers used. Data measured on (A) IN16 at a temperature of $289.2 \mathrm{~K}$ and at a protein concentration of $6.2 \%$, (B) IRIS at a nearly identical concentration of $6.5 \%$ and (C) IN5 at a lower concentration of $4.4 \%$.

\subsection{Internal protein diffusive motions}

The properties of internal diffusive protein motions can be deduced from the broad Lorentzian of the QENS spectra. The $q$-dependence of the line-widths $\Gamma_{2}$ provides information on the relaxation times, jump-diffusion coefficients and residence times of internal protein dynamics on the investigated timescales. Representative line-widths related to internal protein dynamics of MG states of apo-Mb recorded on different neutron spectrometers are shown in Fig. 5.

Line-widths $\Gamma_{2}$ extracted from measurements on the highresolution spectrometer IN16 - see Fig. 5(A) for the case of the MG state of apo-Mb at pD 2 stabilized by $0.1 \mathrm{M} \mathrm{NaCl}$ - were
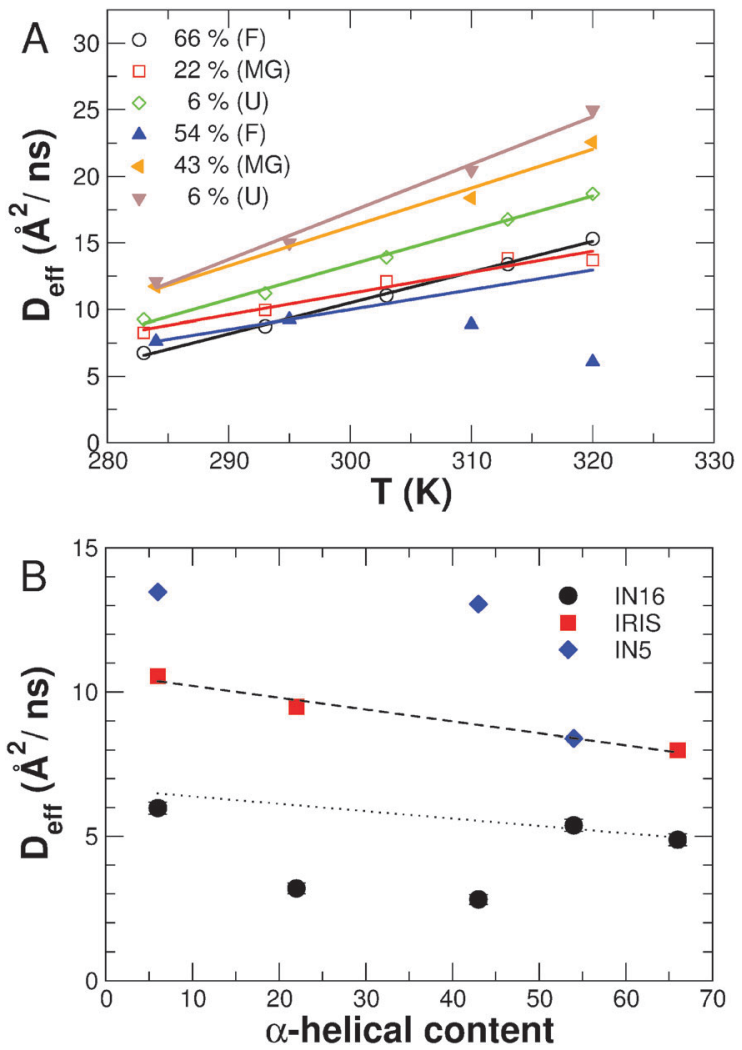

Fig. 4 Effective diffusion coefficients of global protein diffusion of the investigated structural states of apo- and holo-Mb. Error bars are within the symbols. (A) Data obtained on the spectrometers IRIS (empty symbols) and IN5 (filled symbols) as a function of temperature. The $\alpha$-helical content of the native folded (F), partially folded molten globule (MG), and unfolded (U) states is given in the legend. (B) Effective diffusion coefficients as a function of the degree of $\alpha$-helical content at a temperature of $289.2 \mathrm{~K}$. The diffusion coefficients of IRIS and IN5 shown in (A) were linearly interpolated to a temperature of $289.2 \mathrm{~K}$ to allow a direct comparison with IN16 data. The dashed line is a linear fit to the IRIS and IN5 data excluding the more diluted solutions measured on IN5. The dotted line shows the scaled linear fit (dashed line) such that the IN16 value of holo-Mb at $66 \% \alpha$-helical content is matched. The scaled linear fit agrees with the IN16 results of the acid denatured and native apo-Mb states, but reveals deviations of the MGs due to gel formation.

found to be independent of the scattering vector $q$ indicative of a localised dynamic process. Fig. 5(A) depicts the unbiased fit results, without imposing any $q$-dependence. The IN16 QENS spectra were consequently described by a global fitting approach using a $q$-independent line-width $\Gamma_{2}$. The corresponding relaxation times $\tau=\hbar / \Gamma_{2}$ as a function of the $\alpha$-helical content of the protein are given in Fig. $6(\mathrm{~A})$. We find a relaxation time of $\tau=200 \mathrm{ps}$ for all samples being independent of the secondary structure content of the protein. The observed slow dynamic process could be, for example, attributed to the reorientational motions of buried groups in the protein or the slow rotational motions of side-chains. The slow relaxation time of apo-and holo-Mb determined is similar to the previous results on the dynamics of alcohol dehydrogenase $(\tau=160 \mathrm{ps}){ }^{39}$ the LOV photoreceptor protein SB1 in the dark- and light-adapted states ( $\tau=170$ and $150 \mathrm{ps}$, respectively), ${ }^{40}$ but appears to be larger than that of the structurally related haemoglobin in red blood cells 

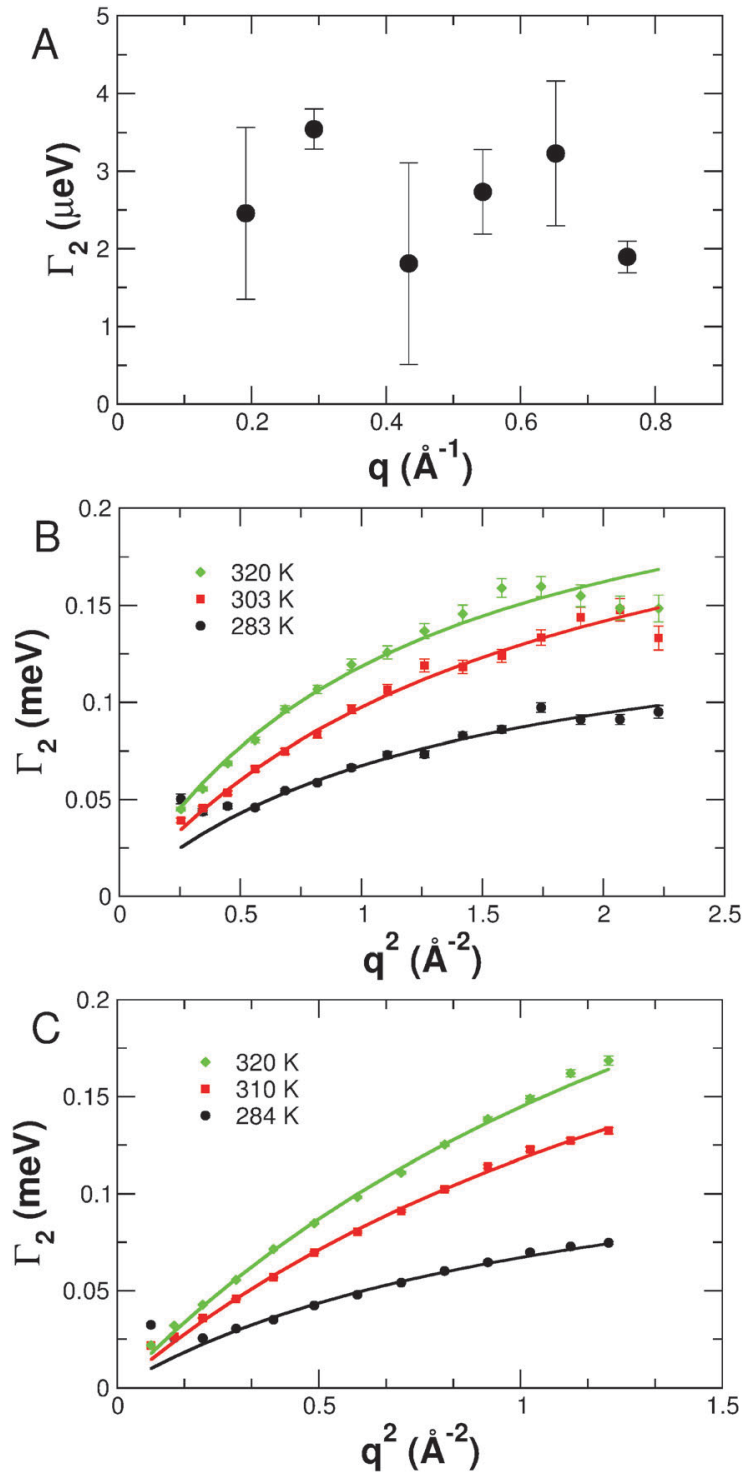

Fig. $5 \mathrm{HWHM} \Gamma_{2}$ of internal protein dynamics of exemplary samples measured on the spectrometers used. Apo-Mb at pD 2 with $0.1 \mathrm{M} \mathrm{NaCl}$ (22\% $\alpha$-helical content) measured on (A) IN16 and (B) IRIS. (C) Apo-Mb at pD 4 ( $43 \% \alpha$-helical content) measured on IN5.

( $\tau=121 \mathrm{ps}),{ }^{33}$ or also of the membrane protein bacteriorhodopsin $(\tau=120 \mathrm{ps}){ }^{41}$

The $\Gamma_{2}$ recorded on IRIS and IN5 of the apo-Mb states showed a $q$-dependent behaviour and could be described analytically by a jump-diffusion mechanism. Fits were done in the $q^{2}$-range of 0.21 $1.12 \AA^{-2}$ for IN5 data and $0.56-2.23 \AA^{-2}$ for IRIS data according to

$$
\Gamma_{2}(q)=\frac{D q^{2}}{1+D q^{2} \tau_{0}},
$$

with the jump-diffusion coefficient $D$ and the residence time $\tau_{0}$

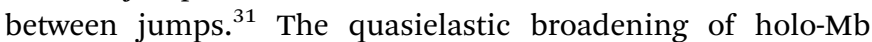
could not be interpreted due to insufficient statistics. The line-widths $\Gamma_{2}$ of the MG states at pD 2 stabilized by $0.1 \mathrm{M} \mathrm{NaCl}$ (22\% $\alpha$-helical content) and at pD 4 (43\% $\alpha$-helical content) determined on IRIS and IN5 are shown in Fig. 5(B) and (C)
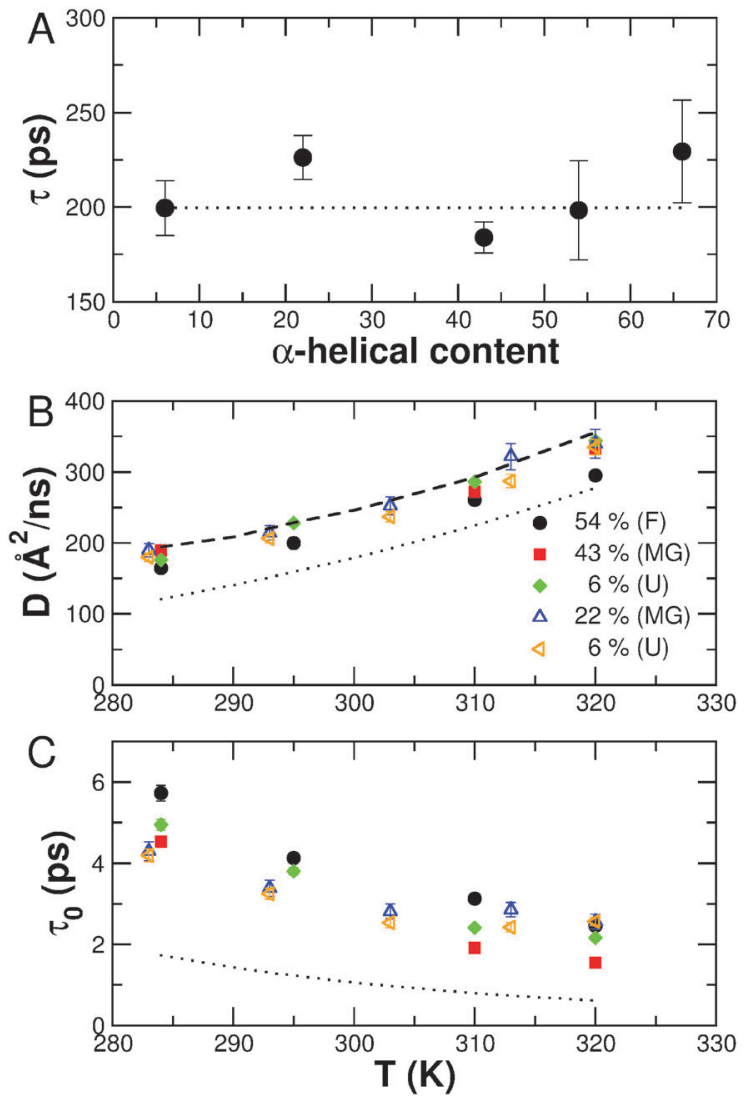

Fig. 6 Information on internal diffusive protein dynamics as deduced from the line-shape analysis of the QENS broadening. (A) Average relaxation times as a function of the $\alpha$-helical content determined on IN16 at a temperature of $289.2 \mathrm{~K}$. The dotted line indicates an average value of $200 \mathrm{ps}$. (B) Diffusion coefficients and (C) residence times of apo-Mb measured on IRIS (empty symbols) and IN5 (filled symbols). The $\alpha$-helical content of the native folded (F), partially folded molten globule (MG), and unfolded (U) states is given in the legend. The dotted lines in (B) and (C) are literature values of $\mathrm{D}_{2} \mathrm{O}$ solvent. ${ }^{44}$ The dashed line in (B) are diffusion coefficients of $\mathrm{H}_{2} \mathrm{O}$ solvent. ${ }^{43}$

together with fits according to eqn (8). The constant plateau of the HWHM at the smallest $q^{2}$-values of the data at the lowest temperature shown is caused by confinement effects of internal dynamics. $^{42}$ The determined jump-diffusion coefficients $D$ are plotted in Fig. 6(B) and the residence times between jumps $\tau_{0}$ in Fig. 6(C). The diffusion coefficients of $\mathrm{H}_{2} \mathrm{O}$ and $\mathrm{D}_{2} \mathrm{O}$ bulk solvents ${ }^{43,44}$ are also plotted in Fig. 6(B) and the residence times of $\mathrm{H}_{2} \mathrm{O} / \mathrm{D}_{2} \mathrm{O}$ in Fig. $6(\mathrm{C})$. The residence times of $\mathrm{H}_{2} \mathrm{O}$ and $\mathrm{D}_{2} \mathrm{O}$ are reported to have similar values. ${ }^{44}$

The observed type of motions on the picosecond time-scale has been attributed to rotational and librational motions of amino acid side-chains along the $\mathrm{C}-\mathrm{C}$ bonds. ${ }^{45}$ More recently, we have attributed the observed dynamics to the jumps of aminoacid side-chains mostly located on the surface of the protein. ${ }^{39,46}$ We find no significant variations of the jump-diffusion coefficients of apo-Mb between the different folded states. The diffusivity of internal protein motions on the picosecond time-scale agrees well with the values of $\mathrm{H}_{2} \mathrm{O}$ bulk solvent. The diffusion coefficients of $\mathrm{D}_{2} \mathrm{O}$ buffer, which was used as solvent for the neutron scattering experiments, are significantly lower than those of internal protein 
dynamics. This is also consistent with the results found for other proteins (see e.g. ref. 47 and references therein). This demonstrates that internal diffusive motions in proteins on the picosecond timescale are governed by H-bonding and depend weakly on the secondary structure content. However, significant variations are observed concerning the residence times of internal protein dynamics. The $\tau_{0}$ of all protein samples are clearly larger than the values of bulk solvent. At low temperatures, the residence times of the folded apo-Mb seems to be increased as compared to the MGs and the unfolded states. The observation indicates that a larger secondary structure content and a higher local compactness of the structure confine an amino-acid residue in its location, and increases the time the side-chain spends in its initial position. This behaviour is also visible in the measured root mean square displacements (RMSDs) (vide infra).

Activation energies $E_{\mathrm{a}}$ were determined from the temperature dependence of the jump-diffusion coefficients and residence times by using the Arrhenius equation. The $E_{\mathrm{a}}$ values determined are summarized in Table 2.

Within the statistical errors the activation energy extracted from the jump-diffusion coefficients is similar for all the investigated structural states (mean value of $12.5 \pm 0.7 \mathrm{~kJ} \mathrm{~mol}^{-1}$ ) measured on both neutron spectrometers. The $\tau_{0}$ at the two highest temperatures determined on IRIS might be overestimated, which changes the activation energies to smaller values. We find larger deviations of the $E_{\mathrm{a}}$ determined from the residence times of the different samples: using the data of IN5 an average value of $19.6 \pm 3.2 \mathrm{~kJ} \mathrm{~mol}^{-1}$ is obtained, while for the proteins measured on IRIS we find an average value of $10.4 \pm 0.4 \mathrm{~kJ} \mathrm{~mol}^{-1}$ in the whole temperature range $\left(17.0 \pm 0.8 \mathrm{~kJ} \mathrm{~mol}^{-1}\right.$ in the restricted temperature range of 283-303 K). The determined values are well in the range of activation energies of internal protein dynamics in haemoglobin ( $9.5 \mathrm{~kJ} \mathrm{~mol}^{-1}$ from jump-diffusion coefficients at $100 \mu \mathrm{eV}$ instrumental resolution $)^{46}$ and free and inhibited acetylcholinesterase (9.4 and $14.5 \mathrm{~kJ} \mathrm{~mol}^{-1}$ from MSDs at $50 \mu \mathrm{eV}$ resolution $)^{48}$ or heat denatured bovine serum albumin (12 and $18 \mathrm{~kJ} \mathrm{~mol}^{-1}$ from the residence times of side-chain and backbone dynamics, respectively, at $3.5 \mu \mathrm{eV}$ resolution). ${ }^{47}$

\subsection{Localized internal protein dynamics}

Information about the amplitude of internal protein dynamics was obtained from the EISF. The measured EISF could be described analytically according to ${ }^{49}$

$$
A_{0}(q)=\mathrm{e}^{-\left\langle x^{2}\right\rangle q^{2}}(1-p)+p
$$

where $\left\langle x^{2}\right\rangle$ is the MSD of internal protein dynamics and $p$ the fraction of $\mathrm{H}$-atoms that move slowly and appear to be immobile within the accessible observation time of the neutron spectrometer. A compilation of EISFs measured on the instruments IRIS and IN5 with fits according to eqn (9) is presented in Fig. 7.

Due to lower counting statistics, the EISF could not be determined accurately from data measured on the highresolution spectrometer IN16. The obtained RMSD $\sqrt{\left\langle x^{2}\right\rangle}$ and the fractions of immobile $\mathrm{H}$-atoms $p$ are plotted in Fig. 8. It is important to stress that the RMSD and $p$ values depend on the energy resolution of the neutron spectrometer. Therefore, a quantitative comparison is strictly only applicable for data sets recorded on neutron spectrometers with the same resolution. The RMSDs extracted from measurements on IN5 are found to be larger than those obtained on IRIS, which is expected, as the energy resolutions of both instruments are different (12 vs. $18 \mu \mathrm{eV}$ FWHM). On IN5, lower protein concentrations have been measured compared to IRIS, which might point out that molecular fluctuations are suppressed at the investigated concentrations on IRIS. The strong increase in the RMSD going from IRIS to IN5 is nevertheless unexpected as no significant molecular crowding interactions are expected for all the measured protein concentrations, which might influence internal molecular dynamics.

The folded apo- and holo-Mb conformations are found to be significantly less flexible than the partially and unfolded conformations, which is in agreement with the previous results on the fast dynamics on the time scale of a few picoseconds. ${ }^{21} A$ priori, one would assume that the fully unfolded states of apo$\mathrm{Mb}$ at pD 2 would be more flexible than the partially folded states of apo-Mb at pD 4 and at pD 2 with $0.1 \mathrm{M} \mathrm{NaCl}$. The experimental data obtained from both experiments show that this is not the case on the accessible time scales of IRIS and IN5, with the MGs states having nearly similar or even higher flexibility than the acid denatured state. The slope of RMSD $v s$. temperature is also flatter for the acid denatured state than the MG states being indicative of stronger internal forces in the fully unfolded protein. An explanation could be that repulsive interactions of charged amino-acid residues in the acid denatured state restrict molecular motions to smaller amplitudes and also lead to stronger internal forces. Less charged residues at $\mathrm{pD} 4$ or a smaller screening length due to the $0.1 \mathrm{M} \mathrm{NaCl}$ content would therefore result in larger amplitudes of motions and a softer structure, albeit the secondary structure content is larger in the MGs than in the acid denatured state.

Table 2 Activation energies of internal protein dynamics

\begin{tabular}{|c|c|c|c|c|}
\hline Spectrometer & Sample & $\alpha$-Helical content (\%) & $E_{\mathrm{a}}$ from $D\left(\mathrm{~kJ} \mathrm{~mol}^{-1}\right)$ & $E_{\mathrm{a}}$ from $\tau_{\mathrm{o}}\left(\mathrm{kJ} \mathrm{mol}^{-1}\right)$ \\
\hline \multirow[t]{3}{*}{ IN5 } & apo-Mb pD 6 & 54 & $12.3 \pm 0.4$ & $17.2 \pm 0.8$ \\
\hline & apo-Mb pD 4 & 43 & $11.7 \pm 0.2$ & $23.2 \pm 0.6$ \\
\hline & apo-Mb pD 2 & 6 & $13.6 \pm 0.3$ & $18.3 \pm 0.7$ \\
\hline \multirow[t]{2}{*}{ IRIS } & apo-Mb pD $20.1 \mathrm{M} \mathrm{NaCl}$ & 22 & $12.5 \pm 0.8$ & $10.6 \pm 1.0^{a}\left(17.6 \pm 3.9^{b}\right)$ \\
\hline & apo-Mb pD 2 & 6 & $12.5 \pm 1.4$ & $10.1 \pm 1.6^{a}\left(16.4 \pm 5.6^{b}\right)$ \\
\hline
\end{tabular}

${ }^{a}$ Determined over whole temperature range. ${ }^{b}$ Limited temperature range $283-303 \mathrm{~K}$. 

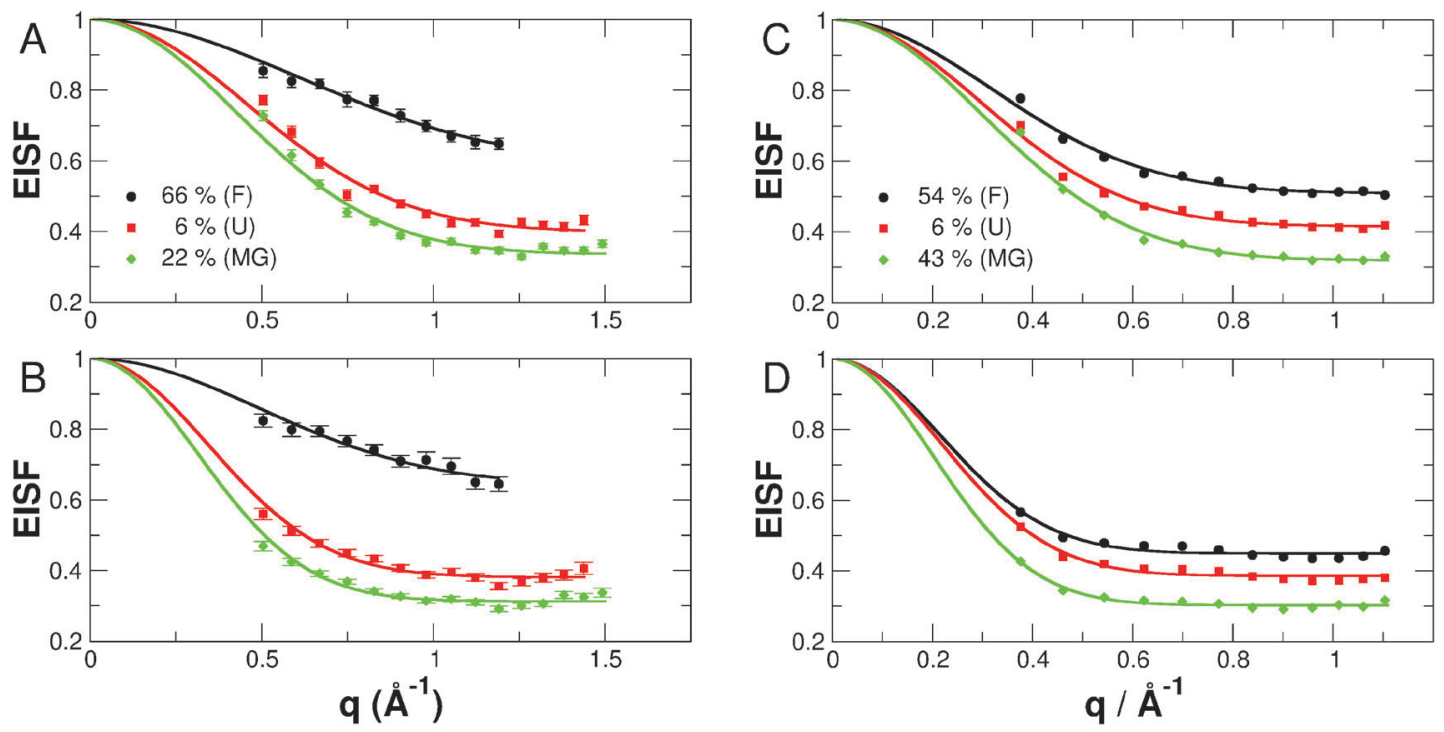

Fig. 7 EISF determined on (A) and (B) IRIS, (C) and (D) IN5. The sample temperatures are (A) $284 \mathrm{~K},(\mathrm{~B}) 320 \mathrm{~K},(\mathrm{C}) 283 \mathrm{~K}$ and (D) $313 \mathrm{~K}$. The $\alpha$-helical content of the native folded (F), partially folded molten globule (MG), and unfolded (U) states is given in the legend.
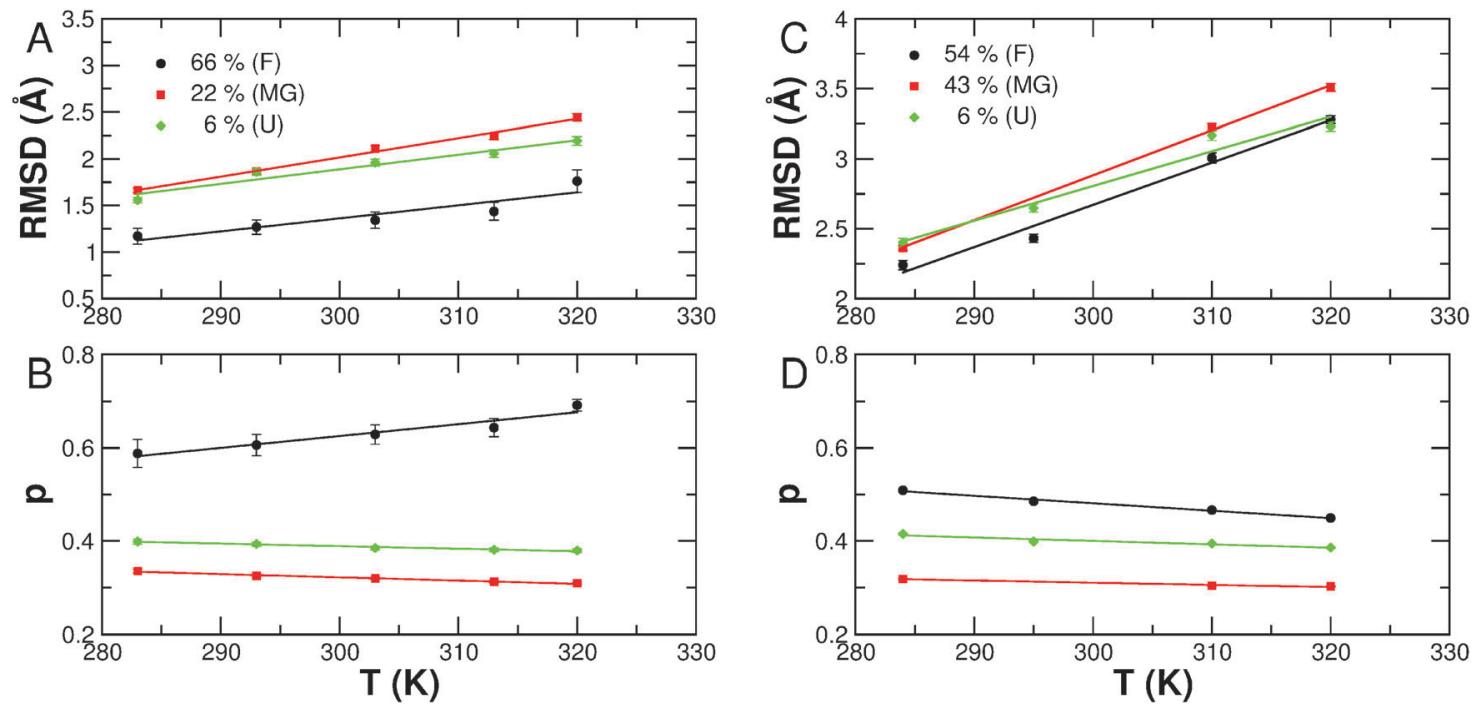

Fig. 8 RMSD and fraction of immobile H-atoms $p$ determined on IRIS and IN5. (A and B) The results obtained on IRIS. (C and D) Quantities measured on IN5. The $\alpha$-helical content of the native folded (F), partially folded molten globule (MG), and unfolded (U) states is given in the legend. The solid lines are linear fits to guide the eye.

The fraction of immobile H-atoms $p$ is attributed to slow moving residues that are located in the hydrophobic core of the protein being shielded from the solvent, ${ }^{21,39}$ and we find general features of the measured samples being independent of the energy resolution of the instruments. For all the investigated conformations of apo-Mb, we find a decrease of $p$ with temperature, which is expected, as higher temperature enhances internal protein dynamics. With regard to holo-Mb, $p$ increases with temperature, which might be related to the fact that the EISF could be determined only in a smaller scattering vector range than for the apo-Mb samples, and the extracted $p$-values for holo-Mb are slightly too large at higher temperatures. Therefore, we discuss only the $p$-values averaged over all temperatures.
The folded conformations of holo- and apo-Mb are characterised by the largest $p$-values. We find average values of $p=0.63$ for holo-Mb and $p=0.48$ for apo-Mb at $\mathrm{pD} 6$. For the acid denatured state, quasi-identical values of $p=0.39$ and 0.40 are determined from IRIS and IN5 data, respectively. This observation is in agreement with the molecular picture that the removal of the heme-group allows water molecules to enter into the heme-pocket, ${ }^{50}$ thus increasing the solvent accessible surface area of the protein. Denaturation of the protein destabilises the hydrophobic core of the folded protein and allows solvent molecules to interact with the otherwise shielded hydrophobic residues. On the other hand, the MG states are characterised by smaller fractions of immobile $\mathrm{H}$-atoms than the unfolded state. 
The average values are nearly identical for both MG states with $p=0.32$ for apo-Mb at $\mathrm{pD} 2$ with $0.1 \mathrm{M} \mathrm{NaCl}$ and $p=0.31$ for apo-Mb at $\mathrm{pD}$ 4. Although the MGs of apo-Mb were found to have a larger secondary structure content and a higher compactness than the acid denatured state, ${ }^{21}$ the experimental data concerning their dynamic properties show that they are highly dynamic objects as also observed before for pressure induced MG states of acetylcholinesterase. ${ }^{51}$ The measured fractions of immobile $\mathrm{H}$-atoms point out that solvent molecules can penetrate into the hydrophobic core of the MG states facilitating fast dynamics on the picosecond time-scale.

\subsection{Conformational entropy of the protein and of the hydration layer}

The knowledge of RMSDs allows the determination of the difference in conformational entropy $\Delta S_{\text {conf }}$ between two protein conformations. In this study, we consider the acid denatured state of apo-Mb at pD 2 as a reference of the unfolded state of apo-Mb. The difference in conformational entropy $\Delta S_{\text {conf }}$ is then given as ${ }^{52,53}$

$$
\Delta S_{\mathrm{conf}}(T)=3 R \ln \sqrt{\frac{\left\langle x_{\mathrm{u}}^{2}\right\rangle(T)}{\left\langle x_{\mathrm{f}}^{2}\right\rangle(T)}},
$$

where $\left\langle x_{\mathrm{u}}{ }^{2}\right\rangle(T)$ and $\left\langle x_{\mathrm{f}}{ }^{2}\right\rangle(T)$ are the MSDs of the unfolded and folded conformations at temperature $T$, respectively, and $R=8.3144 \mathrm{~J} \mathrm{~mol}^{-1} \mathrm{~K}^{-1}$ is the gas constant. The $\Delta S_{\text {conf }}$ value needs to be interpreted on a per residue basis.

The experimentally determined $\Delta S_{\text {conf }}(T)$ profiles of the partially and fully folded conformations of apo- and holo-Mb are plotted in Fig. 9(A) as a function of temperature. In general, larger $\Delta S_{\text {conf }}$ values are found for more folded conformations. The difference in conformational entropy is close to or below zero for the MG states, which is an obvious result as the RMSDs of the acid denatured state are found to be similar or larger than the corresponding values of the MGs. A remarkable result is the parabolic shape of the $\Delta S_{\text {conf }}(T)$ profiles most clearly visible for the native holo- and apo-Mb states at $\mathrm{pD}$ 6. The parabolic profile is reminiscent of the parabolic shape of the thermodynamic free energy difference $\Delta G(T)=\Delta H-T \Delta S$ between the unfolded and folded states of proteins, where $\Delta H$ is the enthalpic component and $\Delta S$ is the contribution due to entropy. The maxima of $\Delta S_{\text {conf }}(T)$ are shifted to lower temperatures from holo-Mb via apo-Mb at pD 6 to the MG states, which is also the case for the thermodynamic $\Delta G(T)$ profiles. ${ }^{21}$ It is important to note here that $\Delta G, \Delta H$ and $\Delta S$ are thermodynamic quantities that contain the contributions of both the protein and the hydration layer, e.g. $\Delta S_{\text {conf }}$ and $\Delta S_{\text {hydr }}$ for the entropic contributions. The entropy difference $\Delta S(T)$ can be calculated as a function of temperature using the Gibbs-Helmholtz equation

$$
\Delta S(T)=\frac{\Delta H}{T_{\mathrm{m}}}+\Delta C_{\mathrm{p}} \ln \left(\frac{T}{T_{\mathrm{m}}}\right),
$$

where $\Delta C_{\mathrm{p}}$ is the heat capacity and $\Delta H$ the enthalpy at the melting temperature $T_{\mathrm{m}}$ determined from calorimetric measurements.

Thermodynamic parameters were taken from the literature $\left(T_{\mathrm{m}}: 81,61,60{ }^{\circ} \mathrm{C} ; \Delta H: 615,222,63.3 \mathrm{~kJ} \mathrm{~mol}^{-1} \mathrm{~K}^{-1} ; \Delta C_{\mathrm{p}}: 11.9\right.$,
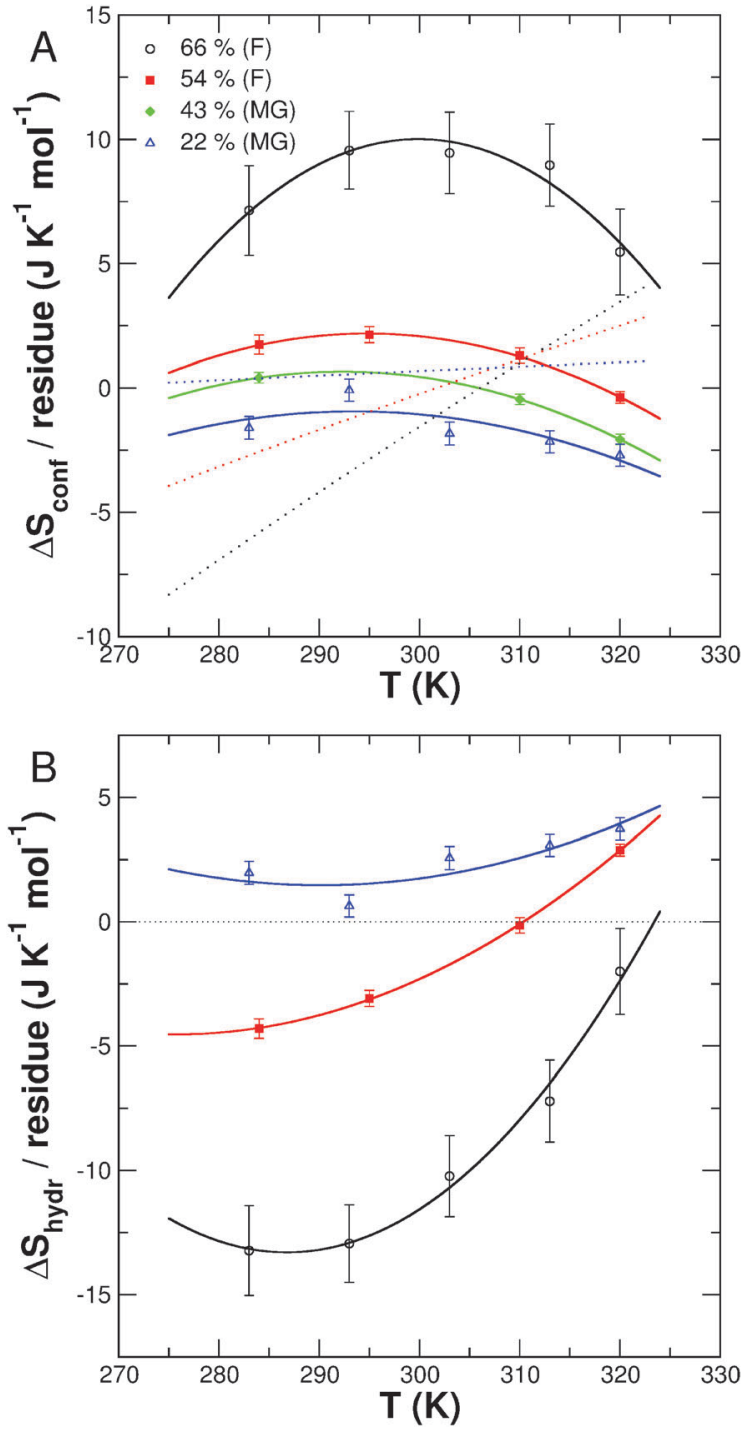

Fig. 9 (A) Difference in conformational entropy $\Delta S_{\text {conf }}$ between the acid denatured state of apo- $\mathrm{Mb}$ and the protein in the investigated partially folded and folded states as obtained from eqn (10). The $\alpha$-helical content of the native folded (F), partially folded molten globule (MG), and unfolded (U) states is given in the legend (empty symbols IRIS, filled symbols IN5 data). The dotted lines are $\Delta S=\Delta S_{\text {conf }}+\Delta S_{\text {hydr }}$ calculated from the thermodynamic parameters $T_{m}, \Delta C_{p}$ and $\Delta H$. (B) Difference in conformational entropy $\Delta S_{\text {hydr }}=\Delta S-\Delta S_{\text {conf }}$ related to water molecules of the hydration shell and protein motions that are out of the time window of the neutron spectrometers. The solid lines in (A) and (B) are polynomial fits of second order and serve as a guide to the eye.

6.5, $0.82 \mathrm{~kJ} \mathrm{~mol}^{-1}$ for holo- and apo-Mb at pH 6 and apo-Mb at pH $20.1 \mathrm{M} \mathrm{NaCl}$, respectively), see ref. 21 and references therein. The values of apo-Mb at $\mathrm{pH} 4$ are not known. The $\Delta S(T)$ curves of folded holo- and apo-Mb (66\% and $54 \%$ $\alpha$-helical content) and of the MG state stabilised by $0.1 \mathrm{M} \mathrm{NaCl}$ (22\% $\alpha$-helical content) are plotted in Fig. 9(A) in comparison to $\Delta S_{\text {conf }}(T)$. The quantities $\Delta S$ and $\Delta S_{\text {conf }}$ are different as $\Delta S_{\text {conf }}$ is derived from the extent of protein fluctuations without the contribution of the hydration shell. The entropy difference between the unfolded and the folded states that is related to 
the contribution of water molecules in the hydration layer of the protein can be determined by

$$
\Delta S_{\text {hydr }}(T)=\Delta S(T)-\Delta S_{\text {conf }}(T) .
$$

The $\Delta S_{\text {hydr }}$ values of the different structural conformations of apo- and holo-Mb are plotted in Fig. 9(B). One should note that the experimentally determined $\Delta S_{\text {hydr }}$ values are not only related to hydration water, but also contain a contribution from slow protein motions that are out of the accessible time window of the neutron spectrometers. Water molecules in the hydration layer of the folded proteins are found to be more disordered and mobile than in the unfolded state, which leads to negative $\Delta S_{\text {hydr }}$ values. With the decreasing degree of protein folding the hydration layer becomes less mobile and for the MG state it is even more ordered than in the unfolded acid denatured apo-Mb, which is visible from the general increase of $\Delta S_{\text {hydr }}$. The physical reason is that water molecules form ordered structures around solvent exposed hydrophobic aminoacids. $^{54}$ With the decreasing $\alpha$-helical content, hydrophobic residues from the protein core become more exposed to solvent, which is also visible from the experimentally determined fractions of immobile $\mathrm{H}$-atoms, and thus induce ordering of the hydration shell. That observation is in agreement with studies on hydration water dynamics of folded and intrinsically disordered proteins that reported reduced motions of the hydration layer around the intrinsically disordered protein. ${ }^{55}$ Interestingly, we observe temperature dependent $\Delta S_{\text {hydr }}$ profiles. At point $\Delta S_{\text {hydr }}\left(T_{0}\right)=0$, the entropic contribution of hydration water of the folded conformation equals that of the hydration layer around the unfolded state and is even smaller at higher temperatures. The intersection temperature $T_{0}$ is shifted to lower temperatures for the less folded apo-Mb states. That behaviour is related to the interplay of two phenomena: (i) melting of the ordered water structures around the hydrophobic residues of the unfolded state at higher temperatures, and (ii) a simultaneous increase of ordered water molecules in the hydration shell of the folded states. An explanation for (ii) might be that water molecules can progressively interact with hydrophobic residues in the core of the folded structures at higher temperatures, which induces ordering of the hydration shell.

\section{Conclusions}

Protein dynamics is of great importance for protein folding, as the polypeptide chain needs to explore the accessible conformational space during the folding transition. In this manuscript, we present an investigation on the global and internal dynamics of $\mathrm{Mb}$ with respect to the secondary structure content of the protein by neutron spectroscopy. The degree of folding is found to have a significant impact on global and internal protein dynamics. An increase in the charge of the macromolecule accelerates global protein short-time diffusion, while a gel-like phase of the MGs at the investigated protein concentrations reduces the overall diffusion on the slow nanosecond time scale. The fully folded protein conformations are found to be significantly less flexible with a higher fraction of immobile $\mathrm{H}$ atoms than the partially and unfolded conformations. The experimental data concerning the internal motions of the MGs demonstrate that they are highly dynamic objects. With regard to internal diffusive protein motions, we find no significant variations of the jump-diffusion coefficients or relaxation times between the different folded states.

The most relevant thermodynamic quantity concerning protein folding that can be determined from the neutron scattering experiments is the difference in conformational entropy $\Delta S_{\text {conf }}$ between two specific structural states. Only few methods are able to determine $\Delta S_{\text {conf }}$ experimentally. NMR, for example, allows determining residue resolved information on the picosecond to nanosecond time scale in terms of the order parameter, which is related to an entropy contribution. Neutron scattering, on the other hand, is a direct method that probes average protein dynamics on the time scale accessible by a neutron spectrometer. As all dynamic processes contribute to $\Delta S_{\text {conf }}$, the average information provided by neutron scattering is actually not a drawback.

In our article, we determined $\Delta S_{\text {conf }}$ between the acid denatured state, which is considered as a reference for the fully unfolded state, and the partially and fully folded conformations as a function of temperature. We find parabolically shaped profiles $\Delta S_{\text {conf }}(T)$ mostly visible for the folded conformations reminiscent of the shape of the thermodynamic free energy difference $\Delta G(T)$. A further important question is, on which time scale the strongest contribution of conformational motions to $\Delta S_{\text {conf }}$ is occurring. In a previous study investigating protein dynamics on the fast time scale of a few picoseconds we found a linear correlation between $\Delta S_{\text {conf }}$ and the secondary structure content, ${ }^{21}$ whereas in the present study we find the $\Delta S_{\text {conf }}$ of the MG states of apo-Mb being similar or below that of the acid denatured states. A general conclusion, therefore, is that the strongest contribution of localised molecular motions detectable by neutron scattering to $\Delta S_{\text {conf }}$ comes from protein fluctuations acting on the fast picosecond time scale.

Thermodynamic experiments allow the determination of $\Delta S$, which contains both the contribution of the protein $\left(\Delta S_{\text {conf }}\right)$ and that of the hydration shell $\left(\Delta S_{\text {hydr }}\right)$. Using thermodynamic parameters from the literature and the experimentally determined $\Delta S_{\text {conf }}$ values as input we could determine the $\Delta S_{\text {hydr }}$ of different folded states of the protein. While the hydration layer of the MG state is found to be slightly more ordered than that of the acid denatured unfolded state, mobile and disordered water molecules in the hydration layer of the folded states make a significant contribution to the entropic stabilisation of the folded structures. At higher temperatures, the hydration layer around the folded states becomes less mobile and more ordered than in the unfolded state, which provides a source for the entropic stabilisation of the unfolded state and facilitates protein unfolding.

\section{Acknowledgements}

The experiments at the ISIS Pulsed Neutron and Muon Source were supported by a beam time allocation from the Science and Technology Facilities Council. We acknowledge the Institut Laue-Langevin for provision of the neutron beam time. 


\section{References}

1 J. D. Bryngelson, J. N. Onuchic, N. D. Socci and P. G. Wolynes, Proteins, 1995, 21, 167-195.

2 V. Daggett and A. R. Fersht, Trends Biochem. Sci., 2003, 28, 18-25.

3 M. Karplus and D. L. Weaver, Protein Sci., 1994, 3, 650-668.

4 C. N. Pace, J. M. Scholtz and G. R. Grimsley, FEBS Lett., 2014, 588, 2177-2184.

5 A. J. Doig and M. J. E. Sternberg, Protein Sci., 1995, 4, 2247-2251.

6 J. Zhang and J. S. Liu, PLoS Comput. Biol., 2006, 2, e168.

7 K. Henzler-Wildman and D. Kern, Nature, 2007, 450, 964-972.

8 R. B. Best, J. Clarke and M. Karplus, J. Mol. Biol., 2005, 349, 185-203.

9 A. M. Stadler, L. Stingaciu, A. Radulescu, O. Holderer, M. Monkenbusch, R. Biehl and D. Richter, J. Am. Chem. Soc., 2014, 136, 6987-6994.

10 H. Frauenfelder, B. H. McMahon and P. W. Fenimore, Proc. Natl. Acad. Sci. U. S. A., 2003, 100, 8615-8617.

11 H. J. Dyson and P. E. Wright, Chem. Rev., 2004, 104, 3607-3622.

12 H. J. Dyson and P. E. Wright, Nat. Struct. Biol., 1998, 5, 499-503.

13 D. Eliezer, J. Yao, H. J. Dyson and P. E. Wright, Nat. Struct. Biol., 1998, 18, 148-155.

14 D. Eliezer and P. E. Wright, J. Mol. Biol., 1996, 263, 531-538.

15 F. Hughson, P. Wright and R. Baldwin, Science, 1990, 249, 1544-1548.

16 D. Eliezer, J. Chung, H. J. Dyson and P. E. Wright, Biochemistry, 2000, 39, 2894-2901.

17 J. Yao, J. Chung, D. Eliezer, P. E. Wright and H. J. Dyson, Biochemistry, 2001, 40, 3561-3571.

18 M. Kataoka, I. Nishii, T. Fujisawa, T. Ueki, F. Tokunaga and Y. Goto, J. Mol. Biol., 1995, 249, 215-228.

19 O. Tcherkasskaya and V. N. Uversky, Proteins, 2001, 44, 244-254.

20 P. C. Aoto, C. Nishimura, H. J. Dyson and P. E. Wright, Biochemistry, 2014, 53, 3767-3780.

21 A. M. Stadler, M. M. Koza and J. Fitter, J. Phys. Chem. B, 2015, 119, 72-82.

22 Neutron Scattering in Biology - Techniques and Applications, ed. J. Fitter, T. Gutberlet and J. Katsaras, Springer Berlin Heidelberg New York, Berlin Heidelberig, 2006.

23 A. K. Covington, M. Paabo, R. A. Robinson and R. G. Bates, Anal. Chem., 1968, 40, 700-706.

24 E. Gasteiger, C. Hoogland, A. Gattiker, S. Duvaud, M. R. Wilkins, R. D. Appel and A. Bairoch, in The Proteomics Protocols Handbook, Humana Press, 2005, pp. 571-607.

25 E. Antonini and M. Brunori, Hemoglobin and myoglobin in their reactions with ligands, North-Holland Pub. Co, Amsterdam, 1971.

26 http:/www.ill.eu/instruments-support/instruments-groups/ yellowbook/.

27 C. J. Carlile and M. A. Adams, Phys. B, 1992, 182, 431-440.

28 Y. Harpaz, M. Gerstein and C. Chothia, Structure, 1994, 2, 641-649.

29 L. R. Murphy, N. Matubayasi, V. A. Payne and R. M. Levy, Folding Des., 1998, 3, 105-118.
30 S. J. Edelstein and H. K. Schachman, J. Biol. Chem., 1967, 242, 306-311.

31 M. Bée, Quasielastic neutron scattering: principles and applications in solid state chemistry, biology, and materials science, Adam Hilger, Bristol and Philadelphia, 1988.

32 F. Roosen-Runge, M. Hennig, F. Zhang, R. M. J. Jacobs, M. Sztucki, H. Schober, T. Seydel and F. Schreiber, Proc. Natl. Acad. Sci. U. S. A., 2011, 108, 11815-11820.

33 A. M. Stadler, L. van Eijck, F. Demmel and G. Artmann, J. R. Soc., Interface, 2011, 8, 590-600.

34 J. Pérez, J. M. Zanotti and D. Durand, Biophys. J., 1999, 77, 454-469.

35 A. M. Gaspar, M.-S. Appavou, S. Busch, T. Unruh and W. Doster, Eur. Biophys. J., 2008, 37, 573-582.

36 A. M. Stadler, I. Digel, G. M. Artmann, J. P. Embs, G. Zaccai and G. Büldt, Biophys. J., 2008, 95, 5449-5461.

37 A. J. Banchio and G. Nägele, J. Chem. Phys., 2008, 128, 104903.

38 M. Hennig, F. Roosen-Runge, F. Zhang, S. Zorn, M. W. A. Skoda, R. M. J. Jacobs, T. Seydel and F. Schreiber, Soft Matter, 2012, 8, 1628.

39 M. Monkenbusch, A. Stadler, R. Biehl, J. Ollivier, M. Zamponi and D. Richter, J. Chem. Phys., 2015, 143, 075101.

40 A. M. Stadler, E. Knieps-Grünhagen, M. Bocola, W. Lohstroh, M. Zamponi and U. Krauss, Biophys. J., 2016, 110, 1064-1074.

41 J. Fitter, R. E. Lechner and N. A. Dencher, Biophys. J., 1997, 73, 2126-2137.

42 F. Volino and A. J. Dianoux, Mol. Phys., 2006, 41, 271-279.

43 A. M. Stadler, J. P. Embs, I. Digel, G. M. Artmann, T. Unruh, G. Büldt and G. Zaccai, J. Am. Chem. Soc., 2008, 130, 16852-16853.

44 Y. Edura and N. Morishima, Nucl. Instrum. Methods Phys. Res., Sect. A, 2005, 545, 309-318.

45 J. M. Zanotti, M. C. Bellissent-Funel and J. Parello, Biophys. J., 1999, 76, 2390-2411.

46 A. M. Stadler, C. J. Garvey, J. P. Embs, M. Koza, T. Unruh, G. Artmann and G. Zaccai, Biochim. Biophys. Acta, 2014, 1840, 2989-2999.

47 M. Grimaldo, F. Roosen-Runge, M. Hennig, F. Zanini, F. Zhang, N. Jalarvo, M. Zamponi, F. Schreiber and T. Seydel, Phys. Chem. Chem. Phys., 2015, 17, 4645-4655.

48 M. Trapp, M. Trovaslet, F. Nachon, M. M. Koza, L. van Eijck, F. Hill, M. Weik, P. Masson, M. Tehei and J. Peters, J. Phys. Chem. B, 2012, 116, 14744-14753.

49 F. Volino, J.-C. Perrin and S. Lyonnard, J. Phys. Chem. B, 2006, 110, 11217-11223.

50 A. M. Stadler, E. Pellegrini, M. Johnson, J. Fitter and G. Zaccai, Biophys. J., 2012, 102, 351-359.

51 J. Marion, M. Trovaslet, N. Martinez, P. Masson, R. Schweins, F. Nachon, M. Trapp and J. Peters, Phys. Chem. Chem. Phys, 2015, 17, 3157-3163.

52 J. Fitter, Biophys. J., 2003, 84, 3924-3930.

53 V. Receveur, P. Calmettes, J. C. Smith, M. Desmadril, G. Coddens and D. Durand, Proteins, 1997, 28, 380-387.

54 P. Ball, Chem. Rev., 2008, 108, 74-108.

55 F. X. Gallat, A. Laganowsky, K. Wood, F. Gabel, L. van Eijck, J. Wuttke, M. Moulin, M. Hartlein, D. Eisenberg, J. P. Colletier, G. Zaccai and M. Weik, Biophys. J., 2012, 103, 129-136. 